\title{
Le suivi des enfants nés par ICSI
}

François Flori

$>$ En novembre 2001, la défenseure des enfants avait saisi le Comité consultatif national d'éthique ( $C C N \varepsilon$ ) sur les dangers de l'injection intracytoplasmique de spermatozoïdes (ICSI). En réponse, dans l'avis $n^{\circ} 75$ intitulé "Questions éthiques soulevées par le développement de l'ICSI» - dont les aspects médicaux reposaient sur deux articles publiées dans médecine/sciences [1,2] - le CCNE faisait remarquer qu'il pouvait sembler «naïf de ne se poser la question éthique de l'ICSI qu'après plusieurs dizaines de milliers de naissances d'enfants. La société semble découvrir que, dans ce domaine, l'innovation scientifique n'a été à l'abri d'un regard critique que par son efficacité reconnue».

\section{Une grande efficacité}

On ne saurait si bien dire: l'ICSI doit d'abord son succès à sa grande efficacité. Mise au point en Belgique en 1991, elle s'est révélée extrêmement efficace: elle a résolu de nombreux cas de stérilité masculine et les centres d'AMP (assistance médicale à la procréation) l'ont adoptée très vite et élargi ses applications dans de très nombreux pays ${ }^{1}$ sans qu'il y ait

\footnotetext{
${ }^{1}$ Le Bulletin épidémiologique hebdomadaire du 14 juin 2011 $\left(n^{\circ} 23-24\right)$ est entièrement dédié aux enjeux et mutations de l'assistance médicale à la procréation. «Assistance médicale à la procréation », 2011, n²3-24, p. 261-284. En effet, on estimait il y a un an qu'il y avait eu environ 4 millions d'enfants nés dans le monde à la suite d'une FIV avec ou sans ICSI. Si I'ICSI n'a débuté qu'en 1992, elle représente aujourd'hui plus de $60 \%$ des tentatives de FIV faites dans la plupart des pays y compris la France (voir l'article de F. Thépot dans le BEH [8]). D'après le bilan de l'Agence de la Biomédecine, il y a eu plus de 8000 enfants nés suite à une ICSI faite cette année là en France.
}

eu d'études probatoires à son utilisation.

\section{Beaucoup plus invasive que la FIV}

Cette technique est pourtant beaucoup plus invasive que la FIV (fécondation in vitro) puisque la fécondation forcée à l'aide d'une micropipette peut introduire dans l'ovocyte des éléments biologiques qui ne pourraient y pénétrer naturellement. De plus, pour pallier la stérilité de certains hommes oligo ou azoospermiques, des spermatozoïdes épididymaires ou testiculaires, sélectionnés sur leur mobilité, sont choisis. Mais dans le cas de spermatozoïdes testiculaires provenant d'hommes dont la spermatogenèse est déficiente, le risque de transmettre à l'enfant une anomalie génétique qui serait à son tour la cause d'une oligo ou d'une azoospermie n'est pas nul. C'est pourquoi, malgré l'efficacité de cette technique, des réticences sont apparues en raison de possibles risques génétiques et épigénétiques. De nombreuses études sur le suivi des enfants nés après ICSI ont alors été effectuées, mais avec des résultats contradictoires. Parmi celles-ci, une étude française sur 368 ICSI pratiquées en raison d'une d'azoospermie obstructive avait montré en 2006 que les fausses couches étaient nettement plus élevées après ICSI utilisant des spermatozoïdes testiculaires [3]. Récemment, viennent d'être publiées deux études, l'une belge [4], I'autre néerlandaise [5], sur des cohortes de plusieurs centaines d'enfants conçus à l'aide de cellules germinales non médecine/sciences,

Éditions EDK,

2, rue Troyon,

92310 Sèvres, France.

fflori@edk.fr
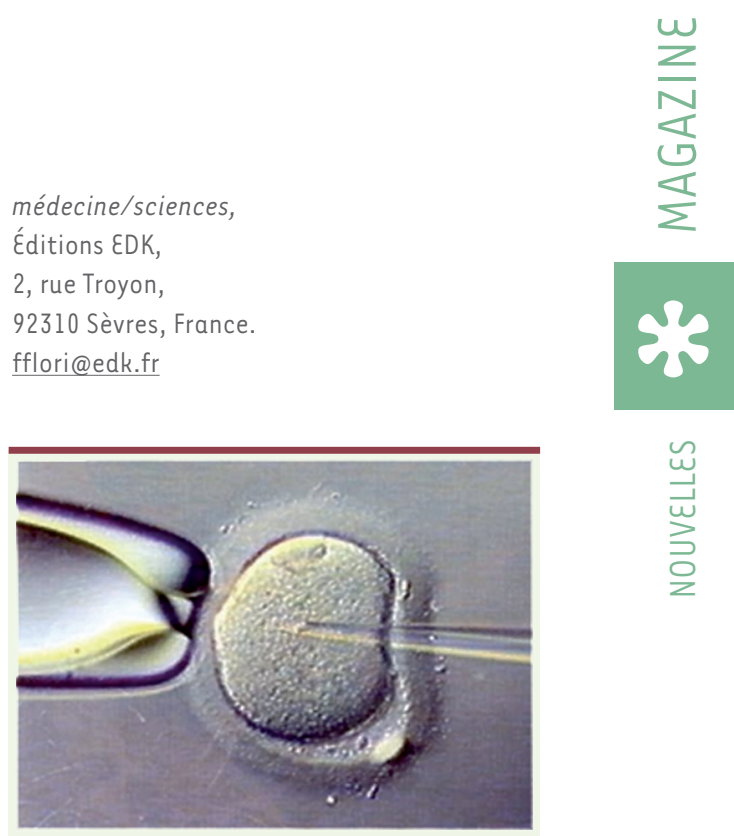

éjaculées. L'étude belge (Université Ziekenhuis, Bruxelles) compare les données néonatales recueillies à partir de questionnaires et d'examens cliniques de 530 enfants nés après fécondation par ICSI/sperme testiculaire, plus 194 enfants nés après ICSI/sperme épididymaire, avec celles de 2516 enfants conçus par ICSI/ sperme éjaculé. Les pourcentages de mortinatalité, de prématurité ainsi que les poids de naissance et les tailles des périmètres crâniens sont comparables. Il n'existe pas d'augmentation du nombre des anomalies majeures (malformations, anomalies chromosomiques). II en va de même dans l'étude néerlandaise (Nimègue, Pays-Bas). Réalisée sur 378 enfants issus d'ICSI/sperme épididymaire, 1192 enfants issus de FIV comparés à 1126 enfants ICSI/sperme éjaculé, elle ne révèle aucune anomalie. Les paramètres péri et néonatals sont analysés et un suivi jusqu'à l'âge de 4 ans de 148 enfants (développement moteur, performance de langage et scores comportementaux) donne des résultats analogues au groupe de référence néerlandais établi en 2002 dans ce pays à partir de l'échelle de 
Bayley modifiée [6]. Peut-être le travail aurait-il été plus complet si une cohorte d'enfants nés de conception naturelle avait été prise en compte dans l'étude.

\section{Des résultats actuels rassurants}

En conclusion, et en se limitant à I'ICSI avec spermatozoïdes épididymaires, bien que cette technique ait a été adoptée d'emblée sans respecter «le principe de précaution », les résultats actuels semblent rassurants sur l'avenir du million d'enfants nés par ICSI dans le monde $[7,8] . \diamond$
Follow-up of the children born by ICSI

\section{REMERCIEMENTS}

À Pierre Jouannet et à Stéphane Viville pour leur relecture du manuscrit.

\section{CONFLIT D'INTÉRÊTS}

L'auteur déclare n'avoir aucun conflit d'intérêts concernant les données publiées dans cet article.

\section{RÉFÉRENCES}

1. Olivennes F, Fanchin R, Righini C, et al. La fécondation in vitro: aujourd'hui et demain. Med Sci (Paris) 2000 ; $16: 316-23$.

2. Guenedal ML, Falquet C, Warter $S$, et al. Les risques liés à l'injection intracytoplasmique du spermatozoïde (ICSI). Med Sci (Paris) 2001 ; $17: 44-53$.
3. Buffat C, Patrat C, Merlet F, et al. CSI outcomes in obstructive azoospermia: influence of the origin of surgically retrieved spermatozoa and the cause of obstruction. Hum Reprod 2006 ; 21 : 1018-24.

4. Belva F, De Schrijver F, Tournaye H, et al. Neonatal outcome of 724 children born after ICSI using nonejaculated sperm. Hum Reprod 2011 ; 26 : 1752-8.

5. Woldrinfh GH, Horvers M, Janssen AJ, et al. Followup of children born after ICSI with epididymal spermatozoa. Hum Reprod 2011 ; 26 : 1759-67.

6. Van der Meulen BF, Ruiter SAJ, Lutje HC, et al. Bayley scales of infant development, $2^{\text {nd }}$ ed. Nederlandse versie, Swets Test Publishers, 2002.

7. Jouannet $P$. L'assistance médicale à la procréation : enjeux et mutations. BEH 2011 ; 23-24 : 261-2.

8. Thépot $F$. Assistance médicale à la procréation : état des pratiques en France. BEH 2011 ; 23-24 : 263-83.

\section{NOUVELLE}

\section{Le chimiotactisme du spermatozoïde est régulé par la fixation de la progestérone sur le canal calcique CATSPER}

Christophe Arnoult, Virginie Pierre, Pierre F. Ray

\author{
Équipe génétique, infertilité et thérapeutique, \\ Laboratoire AGIM, CNRS FRE3405, \\ Université Joseph Fourier de Grenoble, domaine de la Merci, \\ Faculté de médecine, bâtiment Jean Roget, \\ 38700 La Tronche, France. \\ christophe-arnoult@ujf-grenoble.fr
}

> Deux études parues en mars 2011 dans la revue Nature ont réussi à démontrer que le canal CATSPER (cation channel sperm-associated protein) était directement activé par la progestérone [1, 2]. Le canal CATSPER est un canal calcique essentiel à la mobilité du spermatozoïde [3]. Sa structure est similaire à celle des canaux potassiques et forme un pore constitué de quatre sous-unités différentes (CATSPER 1-4), chacune comportant six segments transmembranaires [4]. Au moins deux sous-unités accessoires (CATSPER $\beta$ et $\gamma)$ sont liées à ce canal et sont nécessaires à son fonctionnement $[5,6]$. Ce canal est dépendant du voltage et est fortement activé par une alcalinisation intracellulaire [7]. Il est localisé dans la pièce principale du flagelle du spermatozoïde et est responsable de l'hyperactivation nécessaire à la traversée de la couche protectrice de l'ovocyte appelée zone pellucide (ZP). Des souris déficientes (knock out) pour n'importe quelle sous-unité du canal CATSPER sont totalement stériles, car leurs spermatozoïdes sont incapables de traverser la ZP. Grâce à la technique du patch clamp, les courants ioniques traversant la membrane plasmique du spermatozoïde peuvent être mesurés, et plus particulièrement les courants portés par l'ouverture du canal CATSPER [7]. L'ouverture du canal CATSPER peut également être objectivée par la mesure de la concentration du calcium $\left(\mathrm{Ca}^{2+}\right)$ interne par l'intermédiaire de sondes fluorescentes spécifiques au $\mathrm{Ca}^{2+}$.

\section{Amplification par la progestérone} du courant produit par CATSPER Les auteurs de ces deux publications ont utilisé le patch clamp et la mesure de $\left[\mathrm{Ca}^{2+}\right]$ pour mesurer, soit le courant calcique, soit les variations du $\left[\mathrm{Ca}^{2+}\right]$ intracellulaire dans le spermatozoïde en présence de progestérone. Ils ont ainsi montré que le courant généré par le canal CATSPER est amplifié en réponse à l'exposition à la progestérone. Cet effet est décuplé en présence d'un $\mathrm{pH}$ alcalin. Le courant CATSPER initial et le courant activé par la progestérone sont inhibés par le même antagoniste calcique, NNC55-0396 (ou NCC), inhibiteur des canaux calciques voltage-dépendants de type $T$. Le canal est très sensible à la progestérone car il réagit à des concentrations de progestérone très faibles 